\title{
THE CONCEPT DESIGN OF OBSERVATION CONTROL SYSTEM FOR LIJIANG 2.4-METER TELESCOPE
}

\author{
Chuan-Jun Wang ${ }^{1,2,3}$, Lin-Xie Chen ${ }^{1,2,3}$, Yu-Feng Fan ${ }^{1,2}$, and De-Qing Wang ${ }^{1,2,3}$
}

\begin{abstract}
The Lijiang 2.4-m telescope was built and opened to astronomers since 2008, now there are several instruments installed at the telescope. The control system of the $2.4-\mathrm{m}$ telescope was designed to be operated in robotic mode as the Liverpool telescope, but due to many reasons it didn't achieve robotic mode until now. After we upgraded the fold mirror by adding a rapid instrument exchanging system at the Cassegrain focus in 2012, now we can have up to 9 instruments on the telescope at the same time, but the control system for the instrument is still specific for each instrument. During the observation, change to different control software and operating system for different instrument are needed, which affects the observation significantly. Following the observation control system for few years, we are trying to develop a suitable observation control system for the Lijiang 2.4-m telescope. In this paper, we will introduce the Lijiang observatory, the 2.4-m telescope and its instruments at first, and then discuss the concept design of observation control system for this telescope which will based on the main purpose of improving the observation efficiency.
\end{abstract}

\section{RESUMEN}

El telescopio de 2,4m de diámetro de Lijiang fue construido e inaugurado para las observaciones astronómicas en 2008, contando ahora con varios instrumentos. El sistema de control del telescopio fue diseñado para operar de manera autónoma como el telescopio Liverpool, pero debido a múltiples razones no se ha alcanzado aún el modo de operación robótica. Una vez mejorado el sistema del espejo terciario, ahora es posible tener 9 instrumentos distintos en el telescopio que pueden ser usados en cualquier momento, aunque el sistema de control siga siendo específico para cada instrumento. Durante la noche, puede ser necesario cambiar el sistema de control y el software del propio instrumento según se use un instrumento u otro, lo que afecta a las observaciones. Es por ello por lo que estamos considerando optimizar el sistema de control del telescopio, con el proposito de mejorar la eficiencia del sistema. De todo esto versa este trabajo.

Key Words: instrumentation: miscellaneous

\section{LIJIANG OBSERVATORY OF YNAO}

Lijiang Observatory of YNAO is located at $100^{\circ} 1^{\prime} 48^{\prime \prime}(\mathrm{E}), 26^{\circ} 41^{\prime} 42^{\prime \prime}(\mathrm{N})$, in northwest of Yunnan, China. The observatory was built in 2003 and opened to astronomers in 2008, which has become one of the most important observatories in China. Its altitude is about 3200 meters, and about 600 kilometers away from Kunming (Bai et al. 2010). After the construction of railway, especially the highspeed railway which will be completed next year, it will become more and more convenient for observers travelling to Lijiang Observatory.

Several facilities were installed at Lijiang observatory since 2008 , and there are 8 telescopes at Li-

\footnotetext{
${ }^{1}$ Yunnan Observatories, Chinese Academy of Sciences, Kunming, Yunan,650216, China (wcj@ynao.ac.cn).

${ }^{2}$ Key Laboratory for the Structure and Evolution of Celestial Objects, Chinese Academy of Sciences, Kunming, Yunan, 650216, China.

${ }^{3}$ University of the Chinese Academy of Sciences, Beijing, 100049, China.
}

jiang Observatory nowadays. There are two 2-m class telescopes: a 2.4-m telescope and a 1.8-m telescope, plus several remote control or autonomous telescopes: $60-\mathrm{cm}$ autonomous BOOTES-4 telescope, Taiwan Automated Telescope, 70-cm ChinaThailand remote control telescope, 45-cm YNAOHongkong sky survey telescope and other small telescopes. In the future there will additional telescopes.

The 2.4-m telescope, which was built along with Lijiang observatory, is still the largest common purpose optical telescope in China, and will play more and more important role in the observation of Astronomy. After several years, several kinds of instrument have been installed onto the 2.4-m telescope. The main instrument is still the Yunnan Faint Object Spectrograph and Camera (YFOSC), which can perform photometry and low-medium dispersion spectrum observation. Other instruments like LiJiang Exoplanet Tracker (LiJET), High Resolution Spectrograph (HiRES), Princeton Instruments Ver- 


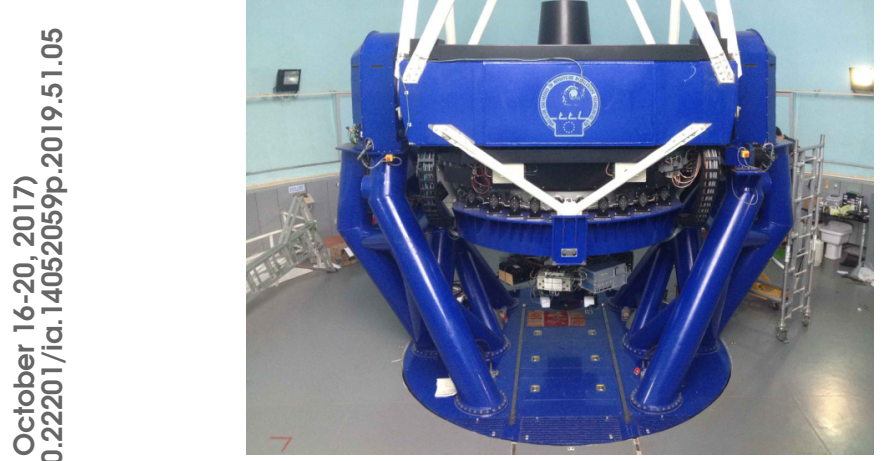

Fig. 1. The structure of Lijiang 2.4-m Telescope.

sArray 1300B CCD (PI CCD) are also installed at the Cassegrain Focus folding position. Thanks to the Rapid Instrument Exchanging System which we installed at 2012, observers can switch to the given instrument they need more quickly and the telescope can also perform several kinds of observations at the single night (Fan et al. 2015).

Based on the improvement of internet connection and update of the network devices, the control system of telescope and all instruments can also be accessed through the internet, which helps the maintaining staff to locate the fault much more quickly even far away. The observers can also perform remote observation with their own computer or laptop through the internet.

\section{LIJIANG 2.4-M TELESCOPE AND MAIN INSTRUMENTS}

Lijiang 2.4-m telescope is an altazimuth reflecting telescope which comprises a Ritchey-Chretien Cassegrain optical design. The diameter of the primary mirror is 2.4 meters which is made of Zerodur crystallite glass provided by Schott company from Germany. It has one Cassegrain focus and two Nasmyth foci, and focusing of the telescope is achieved by moving the secondary mirror. By using the folded mirror, there are eight side ports at the Cassegrain focus which can install different instruments at the same time, which means including in the straight port we have up to nine instruments installed at the Cassegrain focus at the same time. The telescope incorporates motion control system to accurately position the telescope in altitude and azimuth. Addi-

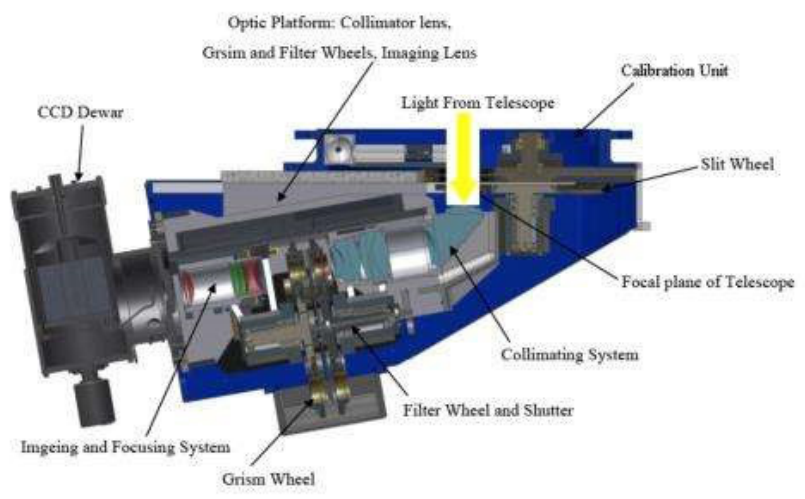

Fig. 2. The structure of YFOSC.

tionally, there is also an autoguider to improve the accuracy of pointing and tracking of the telescope. The structure of the Lijiang 2.4-m telescope is shown in Figure 1.

There are 6 instruments installed on the 2.4-m telescope nowadays, they are YFOSC, LiJET, PI CCD, HiRES, ChiLi (China Lijiang IFU) and EMCCD. Details follow:

\section{1. $Y F O S C$}

YFOSC is a multi-mode instrument based on focal reducer, which can perform photometry, low and medium dispersion spectrum observations, and is the most famous instrument on the 2.4-m telescope. During the night, the observer can attempt different targets and perform different observations depending on the different observing conditions. It is very convenient to change between different filters, grisms and slits. Its structure is shown in Figure 2.

It has five wheels, which are the aperture wheel, the YFUA and YFUB wheels, the filter and grism wheels. All the wheels are controlled by the integrated control system to change between different elements and to perform different observations. YFOSC can obtain a long-slit spectrum (low dispersion) and a cross dispersion spectrum (medium dispersion, up to 10,000). A set of long slits and short slits used for the spectroscopic observations are also available (Zhang et al. 2012).

The CCD for YFOSC is a $2148 \times 4612$ pixel CCD, with the pixel size of $13.5 \mu \mathrm{m} \times 13.5 \mu \mathrm{m}$, which is equal to 0.283 arc seconds in the sky. The readout noise is $3 \mathrm{e}^{-}$, the gain is $0.3 \mathrm{e}^{-}$. For photometry, the field of view is nearly $9.6^{\prime} \times 9.6^{\prime}$. The readout section of the CCD can be set by the size required by the observer to reduce the readout time and improve the observing efficiency. The observer can also using binning to reduce the readout time. 
TABLE 1

YFOSC LONG SLITS

\begin{tabular}{lccc}
\hline No. & Size/um & Sky Angle(") & Pixels \\
\hline L_slit1 & 54 & 0.58 & 2.0 \\
L_slit2 & 74 & 0.79 & 2.8 \\
L_slit3 & 93 & 1.00 & 3.5 \\
L_slit4 & 112 & 1.20 & 4.2 \\
L_slit5 & 140 & 1.50 & 5.3 \\
L_slit6 & 168 & 1.80 & 6.4 \\
L_slit7 & 233 & 2.50 & 8.9 \\
L_slit8 & 480 & 5.05 & 17.7 \\
L_slit9 & 940 & 10.1 & 35.3 \\
\hline
\end{tabular}

TABLE 2

YFOSC SHORT SLITS

\begin{tabular}{lcc}
\hline No. & Size/um & Sky Angle(") \\
\hline S_slit1 & $54 \times 500$ & $0.58 \times 5.37$ \\
S_slit2 & $74 \times 500$ & $0.79 \times 5.37$ \\
S_slit3 & $100 \times 500$ & $1.07 \times 5.37$ \\
S_slit4 & $140 \times 500$ & $1.50 \times 5.37$ \\
S_slit5 & $460 \times 500$ & $4.94 \times 5.37$ \\
S_slit6 & $940 \times 500$ & $10.1 \times 5.73$ \\
\hline
\end{tabular}

\subsection{PI $C C D$}

PI CCD is the first scientific instrument for the 2.4-m telescope, which was bought from Princeton Instrument, US. It was installed at the straight port of Cassegrain Focus, and moved to the side port after the installation of YFOSC and construction of the Rapid Instrument Exchanging System. It has two sets of filters, which are standard Johnson and SDSS filter sets. The parameters of this camera are shown in Table 3. It performs very good photometry on the 2.4-m telescope, and was the main telescope camera prior to YFOSC.

\subsection{HiRES}

HiRES is a high resolution fiber spectrograph, and has two fibers with diameters of 2.0" and 1.6", which provides a 32,000 and 49,000 resolution. The wavelength coverage of HiRES is $320 \mathrm{~nm}$ to $920 \mathrm{~nm}$. Most of the optical elements of HiRES are mounted on the optical platform which is placed in the spectral room at the grand floor and has very stable temperature and pressure control. The light of the star is transferred by the fiber from the telescope in the dome to the spectral room, and then to the CCD camera. The optical layout of HiRES is show in Fig-
TABLE 3

THE PARAMETERS OF PI CCD

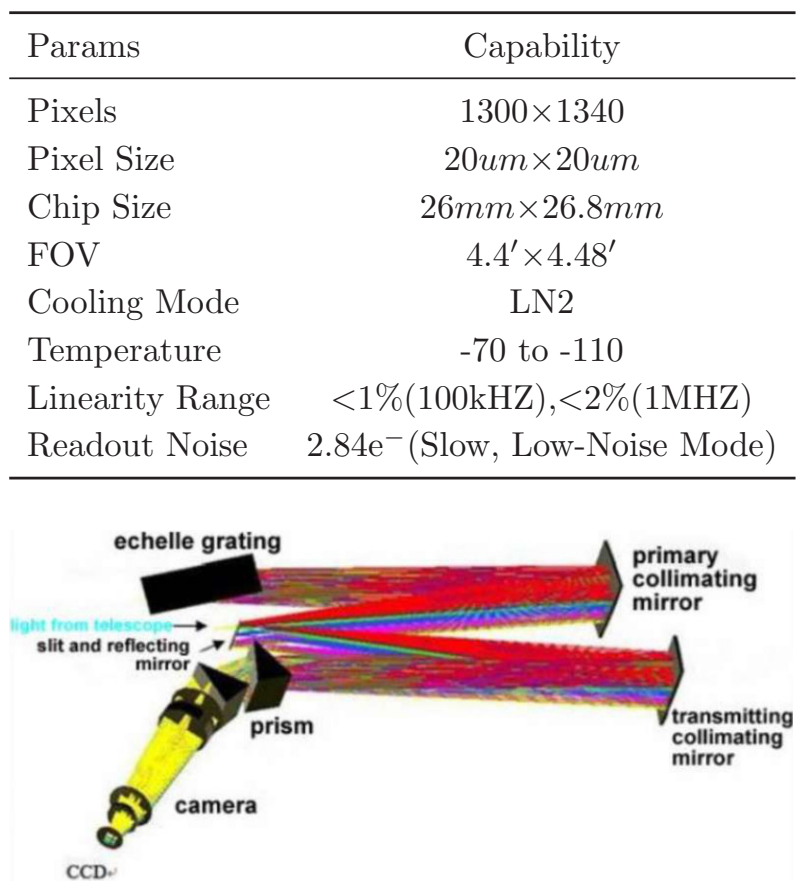

Fig. 3. The optical layout of HiRES.

ure 3 . The $\mathrm{CCD}$ is a $4 K \times 4 K$ detector, and the pixel size is $13.5 \mu m \times 13.5 \mu m$.

\subsection{LiJET}

LiJET is a fiber spectrograph which can be used to provide high-precision radial velocity measurements. It has two observation modes, one is DEM (Direct Echelle Mode) mode, which has a resolution of 30,000 while using the 1.0 " slit, and covers the wavelength between $390 \mathrm{~nm}$ and 1,000nm. Another one is DFDI (Dispersed Fixed-Delay Interferometry) mode, which has a resolution of 18,000 while using the 1.6" slit, and covers the wavelength between $390 \mathrm{~nm}$ and $690 \mathrm{~nm}$. The optical structure of these two modes are show in Figure 4 and Figure 5. In order to detect the high-precision radial velocity, LiJET also has high-precision temperature and pressure control. The temperature and pressure are very stable, which can achieve $0.1 \%$ precision of the variability. The CCD used for LiJET has $4 K \times 4 K$ pixels, the pixel size is $15 \mu m \times 15 \mu m$, the readout noise is $11 e^{-}$, and the gain is $1.37 e^{-}$.

\section{THE CONCEPT DESIGN OF OBSERVATION CONTROL SYSTEM}

The Observation Control System (OCS), also known as Observatory Control System, has become more and more important for the operation 

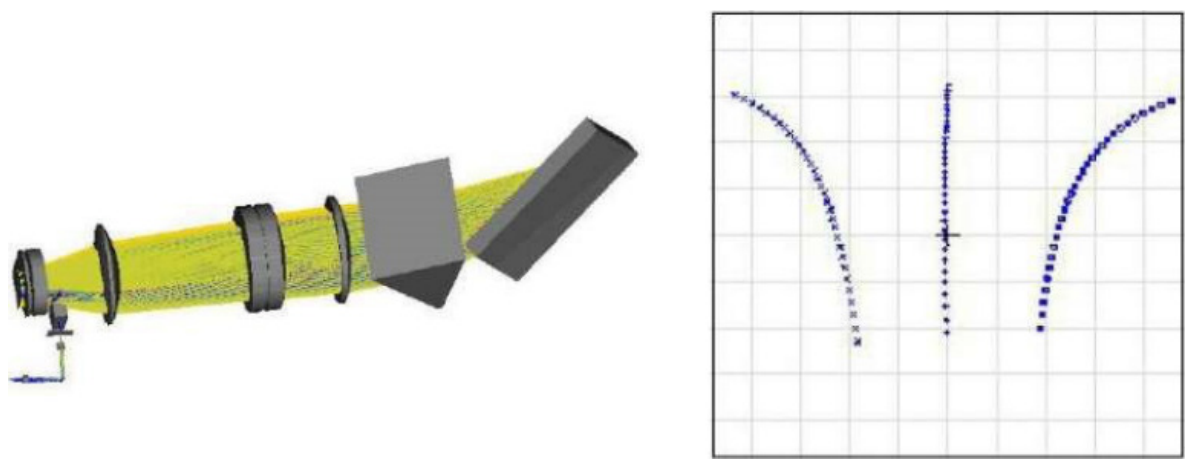

Fig. 4. Layout of the DEM mode (left) and spectra format on the CCD (right).
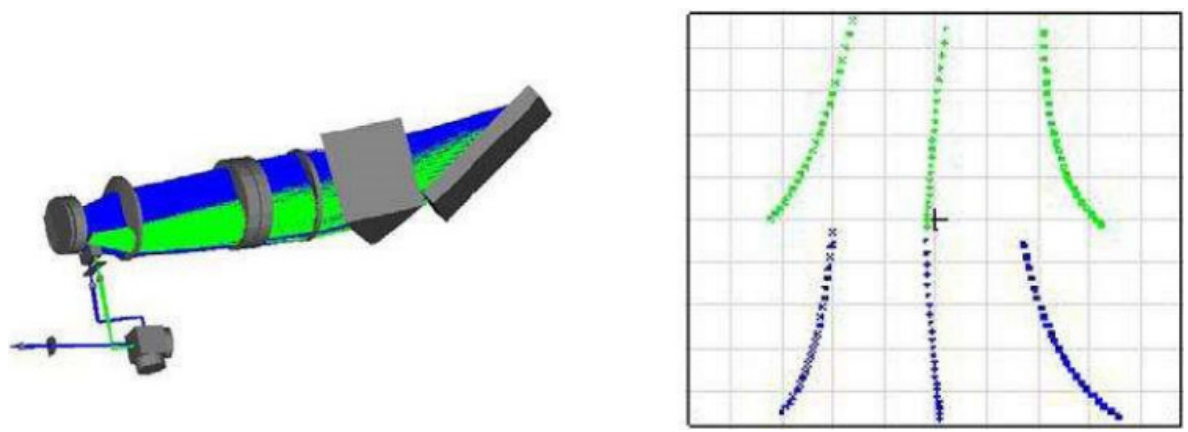

Fig. 5. Layout of Radial Velocity Mode (left) and spectra format on the CCD (right).

and maintainance of one observatory (CastroTirado, 2010; Cui et al. 2013). It is a kind of high-level control system for controling all parts to perform better scheduled observations, including telescope, instruments, data and other systems. The OCS is typically divided into 4 parts, which are Telescope Control System (TCS), Instrument Control System (ICS), Data Handing System (DHS) and Observing Assistant System (OAS), as shown in the Figure 6.

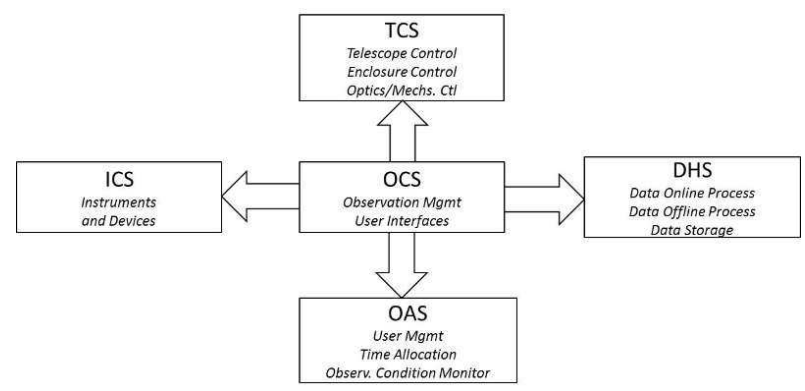

Fig. 6. The different parts of the OCS.

The different parts of OCS are dealing with different parts of the telescope to perform observations:

TCS: Calculates the pointing position of the target, asks the mechanism and optics subsystem to pointing to the target, then tracking it for taking observation.
ICS: Inspects the status of all instruments, and transfers the command from the OCS/observer to the specified instrument. After the exposure is finished, transfers the data to the DHS.

DHS: Does some simple processing of the observation data and prompts some simple results. Does offline processing of the raw data, collecting all metadata into the database. It stores the data into the array and backs up on the remote array.

OAS: Assistant system for the observations, including weather monitor system, cloudy sensor, seeing monitoring, time application and time allocation, observer and proposal management.

OCS: The user interface between observer and all the subsystems. Scheduling all observations of observers whose observation time have been guaranteed by the TAC (Time allocation committee). It gathers all information provided by OAS and executes suitable observation. It receives the quality information of the data from DHS and passes to the observer. It can be considered as the headquarter of the whole system.

The basic structure of the OCS is shown in Figure 7 . 


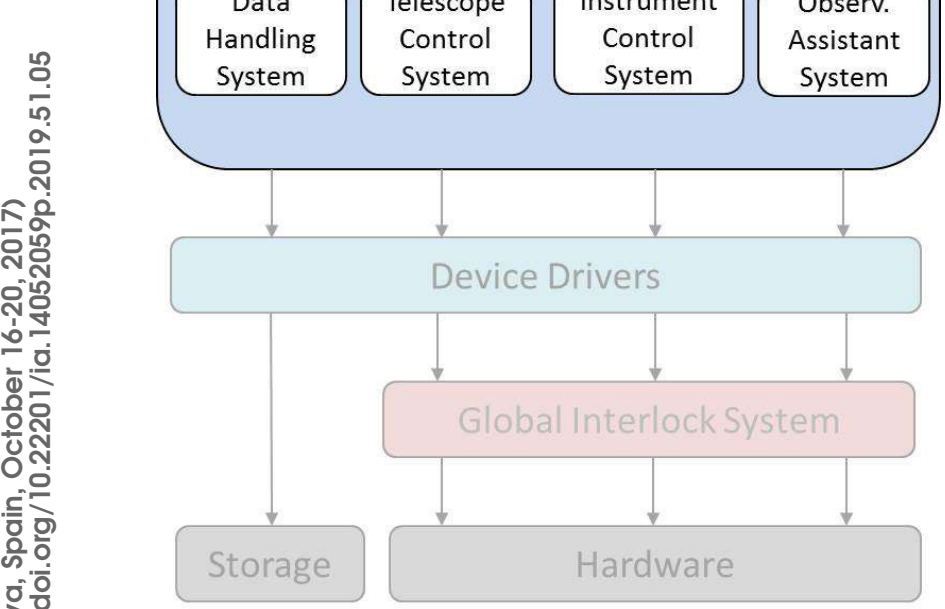

Fig. 7. The basic structure of the OCS.

\subsection{TCS}

For many reasons, neither the source code of the telescope control system for the 2.4-m telescope, nor the whole control system were provided, so it is very difficult for us to re-coding or upgrade the control system. Fortunately, the control system still works fine until now, and we have also got the test interface between the control system and the TCS, which can communicate with the TCS and get the status of the main parts, such as axes, targets, optics, and so on. During the development of OCS, we will just use the interface to communicate with TCS to transfer the command and receive the status back.

\subsection{ICS}

The instruments we used have different control software and are operated under different operating nonstandard control systems as well as different communication interfaces. For the last few years, we have studied the control systems of YFOSC and developed the control software for the 2.4-m telescope under the same control method used in YFOSC (Wang et al. 2013). We also developed the control software for PI CCD under the same control method. The software has been tested successfully and will be applied soon. We are trying to build the control mode based on the YFOSC and PI CCD control software, and then integrated into the structure of ICS. After that, we can develop the common interface for OCS to communicate with ICS.

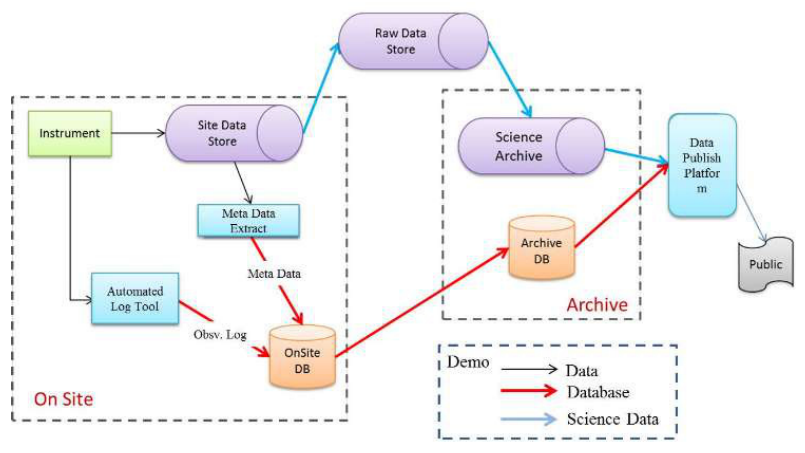

Fig. 8. The structure of DHS.

\section{3. $D H S$}

DHS is responsible for online and offline data process, as well as data storage and backup management. The DHS online process program should work as soon as the exposure is finished, and show up some simple information that refers to the quality of this observation to help observers to adjust their observation strategy. In the daytime, the offline process program will process the raw data, like bias, flat..., and store meta-data of the raw data into the database. At the same time, the raw data will also be stored into the disk array, and backup to remote disk array automatically. The structure of the DHS is shown in Figure 8.

\section{4. $O A S$}

OAS provides all the information that will be needed during the observation, such as the weather condition, cloudy, seeing..., which helps observer to make their decision on targets and observing mode selection. It will also provides the user information, the proposals information and time allocation result, to support OCS on making better observing schedule.

\section{CONCLUSION}

The Lijiang 2.4-m telescope is still the largest common purpose optical telescope in China until now, and has become one of the most important observing instruments in China. After the equipment of many kinds of instruments covering photometry, low-medium dispersion spectrum, high resolution spectrum, lucky imaging and IFU, the $2.4-\mathrm{m}$ telescope can perform many kinds of observations and get valuable observation data. The observation mode and the control system have become the main obstacle for improving the efficiency of this telescope. After the installation of BOOTES-4 (Burst Optical Observer and Transient Exploring System, the 4th Station) on Lijiang Observatory and learning about 
the RTS2 (Remote Telescope System, 2nd Version) from BOOTES-4, we decided to develop the Observation Control System for the Lijiang 2.4-m telescope.

At the current stage, we just present the concept design of the OCS based on the simple study of the current control system of the 2.4-m telescope. We will keep on study the current control system as well as the OCS for other telescopes, and then improve the concept design continuously during the development.

Acknowledgements: We thank the organizers of the Fifth Workshop on Robotic Autonomous Observatory for giving us this chance to introduce our telescope and the system. We thank all of the present and former staff at Lijiang Observatory for their effort on operating and maintaining the Lijiang 2.4-m telescope. This work is supported by the Joint Research Fund in Astronomy (U1631127) under coop- erative agreement between the National Natural Science Foundation of China (NSFC) and Chinese Academy of Science (CAS), and also supported by the Key Laboratory for the Structure and Evolution of Celestial Objects, Chinese Academy of Sciences (CAS).

\section{REFERENCES}

Bai, J. M., \& Liu Z. 2010, 2010, Gaia Follow-up Network for Solar System Objects, 63

Castro-Tirado, A. J. 2010, AdAst, 2010, 570489

Cui, C. Z., Li, J., Cai, X., et al. 2013, PABei, 31, 141

Fan, Y. F., Bai, J. M., Zhang, J. J., Wang, C. J., Chang, L., et al. 2015, RAA, 15, 918

Wang, C. J., Fan, Y. F., \& Yi, W. M. 2013, AR\&T, 10, 378

Zhang, J. J., Fan, Y. F., Chang, L., Wang, C. J., \& Yi, W. M. 2012, AR\&T, 9, 411 